\title{
Nonlinear and memory boundary feedback stabilization for Schrödinger equations with variable coefficients
}

Nawel Abdesselam ${ }^{\mathrm{a}, *}$

${ }^{a}$ Department of Mathematics, Faculty of Sciences Laghouat, University Amar Telidji, Laghouat, Algeria.

\begin{abstract}
In this paper, the boundary stabilization of Schrödinger equations with variable coefficients by nonlinear and memory feedback is considered. The approch adopted uses Riemannian geometry methods and multipliers techniques.@2020 All rights reserved.

Keywords: Schrödinger equation, exponential stabilization, boundary condition of memory type, Riemannian geometry. 2010 MSC: 53B21, 53C21.
\end{abstract}

\section{Introduction}

Let $\Omega$ be an open bounded domain in $\mathbb{R}^{n}$ with boundary $\Gamma:=\partial \Omega$. It is assumed that $\Gamma$ consists of two parts $\Gamma_{0}$ and $\Gamma_{1}$ such that $\Gamma_{0}, \Gamma_{1} \neq \emptyset, \overline{\Gamma_{0}} \cap \overline{\Gamma_{1}}=\emptyset$. We consider the mixed problem for Schrödinger equation

$$
\begin{gathered}
y_{t}-\mathbf{i} A_{y}=0 \quad \text { in } \Omega \times \mathbb{R}_{+}, \\
y(0, x)=y_{0}(x) \quad \text { in } \Omega, \\
y=0 \quad \text { on } \Gamma_{0} \times \mathbb{R}_{+}, \\
\frac{\partial y}{\partial v_{A}}=u \quad \text { on } \Gamma_{1} \times \mathbb{R}_{+},
\end{gathered}
$$

Where

$$
A y=\sum_{i, j=1}^{n} \frac{\partial}{\partial x_{i}}\left(a_{i j}(x) \frac{\partial y}{\partial x_{j}}\right) .
$$

$a_{i j}=a_{j i}$ are $C^{\infty}$ functions in $\mathbb{R}^{n}, \frac{\partial y}{\partial v_{A}}=\sum_{i, j=1}^{n} a_{i j}(x, t) \frac{\partial y}{\partial x_{i}} v_{i}, v=\left(v_{1}, v_{2}, \ldots, v_{n}\right)$ is the unit normal of Ppointing toward the exterior of $\Omega, v_{A}=A v$, and $A=\left(a_{i j}\right)$ is a matrix function. We suppose that

$$
\sum_{i, j=1}^{n} a_{i j}(x) \xi_{i} \overline{\xi_{j}}>\alpha_{1} \sum_{i, j=1}^{n} \xi_{i}^{2} . \forall x \in \Omega, \zeta \in \mathbb{C}^{n}, \zeta \neq 0 .
$$

for some positive constant $\alpha_{1}$.

\footnotetext{
${ }^{*}$ Corresponding author

Email address: nawelabedess@gmail.com (Nawel Abdesselam)
}

doi:https:/ / doi.org/10.31559/glm2020.8.2.1 
Throughout the paper we assume

$$
u=-\int_{0}^{t} k^{\prime}(t-s) \frac{\digamma(|y|)}{|y|}\left(y(s)-y_{t}(t)\right) d s-\frac{1}{2} k y .
$$

where $\quad k: \Gamma_{1} \times \mathbb{R}_{+} \rightarrow \mathbb{R}_{+} \in \mathrm{C}^{2}\left(\mathbb{R}^{+}, \mathrm{L}^{\infty}(\Omega)\right)$, and $\digamma: \mathbb{R}_{+} \rightarrow \mathbb{R}_{+}$is a continuous. we define the corresponding energy functional by

$$
E(t):=\frac{1}{2} \int_{\Omega}\left|\nabla_{g} y\right|_{g}^{2} d \Omega+\int_{\Gamma_{1}}|y|^{2} d \Gamma_{1}+\frac{1}{2} \int_{0}^{t} \int_{\Gamma_{1}} k(t-s) \digamma(|y(s)|)|y(s)| d \Gamma_{1} d s .
$$

The goal of this work is to stabilize the system $(1.1 \mathrm{a}-1.1 \mathrm{~d})$ and $(1.3)$; to find a suitable feedback $u=$ $F\left(x, y_{t}\right)$ such that the energy (1.3) decays to zero exponentially as $t \rightarrow+\infty$ for every solution $y$ of which $\mathrm{E}(0)<+\infty$. The approch adopted uses Riemannian geometry, this method was first introduced into the boundary-control problem by Yao [10] for the exactly controllability of wave equations.

The stabilization of partial differential equations has been considered by many authors.

The asymptotic behaviour of the wave equation with memory and linear feedbacks with constant coefficients has been studied by Guesmia [4], and by Aassila et al [1] in the case nonlinear .This study has been generalised by Chai Guo [2], with variable coefficients by using a very different method, namely, the Riemannian geometry method.

On the other hand, the stabilization of the Schrödinger equation has been studied by Machtyngier Zuazua [9] in the Neumann boundary conditions, and by Cipolatti et al [3] with nonlinear feedbacks, this study has been considered by Lasiecka Triggani[7] with constant coefficients acting in the Dirichlet boundary conditions.

The objective of this work, we consider the boundary stabilization for system $(1.1 \mathrm{a}-1.1 \mathrm{~d})$ and (1.3) with variable coefficients and memory with nonlinear feedbacks by using multipliers techniques.

Our paper is organized as follows. In subsection 1.1, we introduce some notations and results on Riemannian geometry. Our main results are studied in section 2. Section 3 is devoted to the proof of the main results.

\section{Euclidean metric on $\mathbb{R}^{n}$}

Let $\left(x_{1}, \ldots, x_{n}\right)$ be the natural coordinate system in $\mathbb{R}^{n}$. For each $x \in \mathbb{R}^{n}$, denote

$$
X \cdot Y=\sum_{i=1}^{n} \alpha_{i} \beta_{i}, \quad|X|_{0}^{2}=X \cdot X, \quad \text { for } X=\sum_{i=1}^{n} \alpha_{i} \frac{\partial}{\partial x_{i}}, \quad Y=\sum_{i=1}^{n} \beta_{i} \frac{\partial}{\partial x_{i}} \in T_{X} \mathbb{R}^{n} .
$$

For $f \in C^{1}(\bar{\Omega})$ and $X=\sum_{i=1}^{n} \alpha_{i} \frac{\partial}{\partial x_{i}}$, we denote by

$$
\nabla_{0} f=\sum_{i=1}^{n} \frac{\partial f}{\partial x_{i}} \frac{\partial}{\partial x_{i}} \quad \text { and } \quad \operatorname{div}_{0}(X)=\sum_{i=1}^{n} \frac{\partial \alpha_{i}(x)}{\partial x_{i}}
$$

the gradient of $f$ and the divergence of $X$ in the Euclidean metric.

\section{Riemannian metric}

For each $x \in \mathbb{R}^{n}$, define the inner product and the corresponding norm on the tangent space $T_{x} \mathbb{R}^{n}$ by

$$
\begin{gathered}
g(X, Y)=\langle X, Y\rangle_{g}=X \cdot G(X) Y=\sum_{i, j=1}^{n} g_{i j}(x) \alpha_{i} \beta_{j} \\
|X|_{g}^{2}=\langle X, Y\rangle_{g} \quad \text { for } X=\sum_{i=1}^{n} \alpha_{i} \frac{\partial}{\partial x_{i}}, \quad Y=\sum_{i=1}^{n} \beta_{i} \frac{\partial}{\partial x_{i}} \in T_{X} \mathbb{R}^{n}
\end{gathered}
$$


Then $\left(\mathbb{R}^{n}, g\right)$ is a Riemannian manifold with a Riemannian metric $g$. Denote the Levi-Cevita connection in metric $g$ by D. Let $H$ be a vector field on $\left(\mathbb{R}^{n}, g\right)$. The covariant differential DH of $H$ determines a bilinear form on $T_{x} \mathbb{R}^{n} \times T_{x} \mathbb{R}^{n}$. For each $x \in \mathbb{R}^{n}$, by

$$
\operatorname{DH}(X, Y)=\left\langle D_{X} H, Y\right\rangle_{g}, \forall X, Y \in T_{X} \mathbb{R}^{n}
$$

where $\mathrm{D}_{X} \mathrm{H}$ is the covariante derivative of $\mathrm{H}$ with respect to $\mathrm{X}$. The following lemma provides some useful equalities.

Lemme 1.1[Yao, 1999, lemma 2.1].

Let $\mathrm{f}, \mathrm{h} \in \mathrm{C}^{1}(\bar{\Omega})$ and let $\mathrm{H}, \mathrm{X}$ be vector field on $\mathbb{R}^{\mathrm{n}}$. Then with reference to the above notation, we have (i)

$$
\langle\mathrm{H}(\mathrm{x}), \mathrm{A}(\mathrm{x}) \mathrm{X}(\mathrm{x})\rangle_{\mathrm{g}}=\mathrm{H}(\mathrm{x}) \mathrm{X}(\mathrm{x}), \quad \forall \mathrm{x} \in \mathbb{R}^{\mathrm{n}}
$$

(ii) The gradient $\nabla_{\mathrm{g}} \mathrm{f}$ of $\mathrm{f}$ in the Riemannian metric $\mathrm{g}$ is given by

$$
\nabla_{g} f(x)=\sum_{i=1}^{n}\left(\sum_{j=1}^{n} a_{i j}(x) \frac{\partial f}{\partial x_{j}}\right) \frac{\partial}{\partial x_{i}}=A(x) \nabla_{0} f .
$$

(iii)

(vi)

$$
\frac{\partial y}{\partial v_{A}}=\left(A(x) \nabla_{0} y\right) \cdot v=\nabla_{g} y \cdot v
$$

$$
\left\langle\nabla_{g} f, \nabla_{g} H\right\rangle_{g}=\nabla_{g} f(h)=\nabla_{0} f . A(x) \nabla_{0} h
$$

$(v)$

$$
\left\langle\nabla_{g} f, \nabla_{g} H(f)\right\rangle=D H\left(\nabla_{g} f, \nabla_{g} f\right)+\frac{1}{2} \operatorname{div}_{0}\left(\left|\nabla_{g} f\right|_{g}^{2} H\right)(x)-\frac{1}{2}\left|\nabla_{g} f\right|_{g}^{2} \operatorname{div}_{0}(H) \quad x \in \mathbb{R}^{n}
$$

\section{Statement of main result}

To obtain the boundary stabilization of problem $(1.1 \mathrm{a}-1.1 \mathrm{~d})$ and $(1.2)$, the following hyptheses are assumed.

There exists a vector field $\mathrm{H}$ on the Riemannian manifold $\left(\mathbb{R}^{\mathrm{n}}, \mathrm{g}\right)$ such that

$$
\begin{gathered}
\forall X \in T_{X} \mathbb{R}^{n}, a>0,\left\langle D_{X} H, X\right\rangle_{g} \geqslant a|X|_{g}^{2} . \\
H(x) . v<0, \quad \text { on } \Gamma_{0},
\end{gathered}
$$

and

$$
H(x) . v \geqslant 0, \quad \text { on } \Gamma_{1} .
$$

We also assumed the feedback function $\digamma$ satisfies

$$
\digamma(|y|) \leqslant|y|
$$

and $k \geqslant 0$ and $k^{\prime} \leqslant 0$, on $\Gamma_{0} \times \mathbb{R}_{+}$. Moreover,

$$
\begin{gathered}
k(0)=0 \\
\varphi=\inf _{(x, t) \in \Gamma_{0} \times \mathfrak{R}^{+}}\left(-k^{\prime}\right) \neq 0
\end{gathered}
$$




$$
k^{\prime /} \geqslant 0 \text {. }
$$

We have the following result of existence and uniqueness of strong and weak solution to $(1.1 \mathrm{a}-1.1 \mathrm{~d})$ and (1.2) .

Theorem 2.1. For all initial data $y_{0} \in \mathrm{V}=\mathrm{H}_{\Gamma_{0}}^{1}(\Omega)=\left\{\mathrm{y} \in \mathrm{H}^{1}(\Omega) ; \mathrm{y}=0\right.$ on $\left.\Gamma_{0}\right\}$, problem (1.1a - 1.1d) and (1.3) admits a unique global weak solution $\mathrm{y} \in \mathrm{C}\left(\mathbb{R}^{+}, \mathrm{V}\right)$. Furthermore, if $\mathrm{y}_{0} \in \mathrm{H}^{3}(\Omega) \cap \mathrm{H}_{\Gamma_{0}}^{1}(\Omega)$ and $\frac{\partial y_{0}}{\partial v_{\mathrm{A}}}=-\frac{1}{2} \mathrm{ky}(0)$ on $\Gamma_{1}$, then the solution has the regularity

$$
y \in C^{1}\left(\mathbb{R}^{+}, \mathrm{V}\right)
$$

Proof. We can prove, using Galerkin method [6].

Suppose $y$ is the unique global weak solution of problem $(1.1 \mathrm{a}-1.1 \mathrm{~d})$ and (1.2).

Our main result is the following:

Theorem 2.2

Under the hypothesis (1.2)-(1.4) and (2.1)_(2.7).

The problem

$$
\begin{aligned}
y_{t}-\mathbf{i} A y=0 & \text { on } \Omega \\
y=0 & \text { in } \Sigma_{0} \\
\frac{\partial y}{\partial v_{A}}=0 & \text { in } \Sigma
\end{aligned}
$$

and $\mathrm{y}=0$ is the unique solution of the problem.

Then for all given initial data $\mathrm{y}_{0} \in \mathrm{H}_{\Gamma_{0}}^{1}(\Omega)$, there exist two positive constants $\mathrm{M}>0$ and $\omega>0$ such that

$$
E(t) \leqslant M e^{-\omega t} E(0), \quad t>0 .
$$

\section{PROOF OF MAIN RESULT}

For simplicity, we assume that $y$ is a strong solution. By a classical density argument, Theorem 2.2 still holds for a weak solution.

Lemma 3.1. The energy defined by (1.4) is decreasing and satisfies

$$
\begin{aligned}
E(T)-E(0)= & -\int_{0}^{T} \int_{\Gamma_{1}} b\left|y_{t}\right|^{2} d \Gamma_{1} d t+\frac{1}{2} \int_{0}^{T} \int_{\Gamma_{1}} k^{\prime}|y|^{2} d \Gamma_{1} d t \\
& -\frac{1}{2} \int_{0}^{T} \int_{0}^{t} \int_{\Gamma_{1}} k^{\prime \prime}(t-s)|y(t)-y(s)|^{2} d \Gamma_{1} d s d t .
\end{aligned}
$$

where $\mathrm{b}=-\int_{0}^{\mathrm{t}} \mathrm{k}^{\prime}(\mathrm{t}-\mathrm{s}) \frac{\digamma(|\mathrm{y}|)}{|\mathrm{y}|} \mathrm{ds}$, and whenever $0<\mathrm{T}<\infty$.

Proof. Differentiating the energy E (.) defined by (1.4) and using Green's second theorem, we have

$$
\begin{aligned}
E^{\prime}(t)= & -\operatorname{Re} \int_{\Gamma_{1}} \mathrm{ky} \overline{y_{\mathrm{t}}} \mathrm{d} \Gamma_{1}-\operatorname{Re} \int_{\Gamma_{1}}\left(\int_{0}^{\mathrm{t}} \mathrm{k}^{\prime}(\mathrm{t}-\mathrm{s}) \rho(\mathrm{y}(\mathrm{s})) \mathrm{ds}\right) \overline{\mathrm{y}_{\mathrm{t}}} \mathrm{d} \Gamma_{1} \\
& +\int_{\Gamma_{1}} \mathrm{~b}\left|\mathrm{y}_{\mathrm{t}}\right|^{2} \mathrm{~d} \Gamma_{1}+2 \operatorname{Re} \int_{\Gamma_{1}} \mathrm{y} \overline{y_{\mathrm{t}}} \mathrm{d} \Gamma_{1}+\int_{0}^{\mathrm{t}} \int_{\Gamma_{1}} k^{\prime}(\mathrm{t}-\mathrm{s}) \digamma(|\mathrm{y}(\mathrm{s})|)|\mathrm{y}(\mathrm{s})| \mathrm{d} \Gamma_{1} \mathrm{~d} s .
\end{aligned}
$$

By the boundary condition, and by the assumption (2.5) and several technics we obtain that

$$
\begin{aligned}
E^{\prime}(t)= & -\int_{\Gamma_{1}} b\left|y_{t}\right|^{2} d \Gamma_{1}+\frac{1}{2} \int_{\Gamma_{1}} k^{\prime}|y|^{2} d \Gamma_{1} \\
& -\frac{1}{2} \int_{0}^{t} \int_{\Gamma_{1}} k^{\prime \prime}(t-s)|y(t)-y(s)|^{2} d \Gamma_{1} d s .
\end{aligned}
$$


This completes the proof of lemma 3.1.

\section{Lemma 3.2.}

For all $0 \leqslant \mathrm{~S}<\mathrm{T}<\infty$ we have

$$
\begin{aligned}
& \int_{S}^{T} \int_{\Gamma_{1}}\left(\frac{\partial y}{\partial v_{A}}\right)^{2} \frac{H . v}{\left|v_{A}(x)\right|_{g}^{2}} \mathrm{~d} \Gamma_{1} \mathrm{~d} s-\int_{S}^{T} \int_{\Gamma_{1}}\left|\nabla_{g} y\right|_{g}^{2} H v d \Gamma_{1} d s \\
& +2 \operatorname{Re} \int_{S}^{T} \int_{\Gamma_{1}}\left(\frac{\partial y}{\partial v_{A}}\right) H(\bar{y}) d \Gamma_{1} d s+\operatorname{Im} \int_{S}^{T} \int_{\Gamma_{1}} y \overline{y_{t}} H v d \Gamma_{1} d s= \\
& 2 \operatorname{Re} \int_{S}^{T} \int_{\Omega} \operatorname{DH}\left(\nabla_{g} y, \nabla_{g} y\right) d \Omega d s-\int_{S}^{T} \int_{\Omega}\left|\nabla_{g} y\right|_{g}^{2} \operatorname{div} \operatorname{Hd} \Omega d s \\
& +\left.\operatorname{Im} \int_{\Omega} y_{t} H(y) d \Omega\right|_{S} ^{T}+\operatorname{Im} \int_{S}^{T} \int_{\Omega} \overline{y_{t}} y d i v H d \Omega d s
\end{aligned}
$$

Proof. We multiply (1.1a) by H. $\nabla \bar{y}$ and intgrate over $] S, T[\times \Omega$ we obtain

$$
\int_{S}^{T} \int_{\Omega} y_{t} H \cdot \nabla \bar{y} d \Omega d s-i \int_{S}^{T} \int_{\Omega} A y H \cdot \nabla \bar{y} d \Omega d s=0
$$

We have

$$
\begin{aligned}
\int_{S}^{T} \int_{\Omega} y_{t} H \cdot \nabla \bar{y} d \Omega d s= & \left.\int_{\Omega} y H \cdot \nabla \bar{y} d \Omega\right|_{S} ^{T}-\int_{S}^{T} \int_{\Omega} y H \cdot \nabla \overline{y_{t}} d \Omega d s . . \\
= & \left.\int_{\Omega} y H \cdot \nabla \bar{y} d \Omega\right|_{S} ^{T}-\int_{S}^{T} \int_{\Gamma} y \overline{y_{t}} H \cdot v d \Gamma d s 3.1 b \\
& +\int_{S}^{T} \int_{\Omega} \overline{y_{t}} d i v(y . H) d \Omega d s .
\end{aligned}
$$

Substituting (3.1b) into (3.1a), we get

$$
\begin{gathered}
\left.\int_{\Omega} y H \cdot \nabla \bar{y} d \Omega\right|_{S} ^{T}-\int_{S}^{T} \int_{\Gamma} y \overline{y_{t}} H \cdot v d \Gamma d s-\mathbf{i}\left[\int_{S}^{T} \int_{\Omega}(A \bar{y} H \cdot \nabla y+A y H \cdot \nabla \bar{y}) d \Omega d s\right] \\
+\int_{S}^{T} \int_{\Omega} \overline{y_{t}} y d i v H d \Omega d s=0 .
\end{gathered}
$$

Hence

$$
\begin{aligned}
2 \operatorname{Re} \int_{S}^{T} \int_{\Omega} A y H \cdot \nabla \bar{y} d \Omega d s & =\left.\operatorname{Im} \int_{\Omega} y H \cdot \nabla \bar{y} d \Omega\right|_{S} ^{T}-\operatorname{Im} \int_{S}^{T} \int_{\Gamma} y \overline{y_{t}} H . v d \Gamma d s \\
& +\operatorname{Im} \int_{S}^{T} \int_{\Omega} \overline{y_{t}} y d i v H d \Omega d s
\end{aligned}
$$

Using (1.13) of lemma 1.1, we rewrite the integral on the left-hand side of (3.1c) as

$$
\begin{aligned}
\int_{S}^{T} \int_{\Omega} A y H \cdot \nabla \bar{y} d \Omega d s & =\int_{S}^{T} \int_{\Gamma} \frac{\partial y}{\partial v_{A}} H(y) d \Gamma d s-\int_{S}^{T} \int_{\Omega} D H\left(\nabla_{g} y, \nabla_{g} \bar{y}\right) d \Omega d s \\
& -\frac{1}{2} \int_{S}^{T} \int_{\Omega} \operatorname{div}\left(\left|\nabla_{g} y\right|^{2} \cdot H\right) d \Omega d s \\
& +\frac{1}{2} \int_{S}^{T} \int_{\Omega}\left|\nabla_{g} y\right|_{g}^{2} \operatorname{div} H d \Omega d s
\end{aligned}
$$


Recalling the boundary condition $(1.1 \mathrm{~b})$, we have on $\Gamma$

$$
y=y_{t}=0 ;\left|\nabla_{g} y\right|_{g}^{2}=\frac{1}{\left|v_{A}(x)\right|_{g}^{2}}\left(\frac{\partial y}{\partial v_{A}}\right)^{2} ; H(y)=\frac{H \cdot v}{\left|v_{A}(x)\right|_{g}^{2}}\left(\frac{\partial y}{\partial v_{A}}\right) .
$$

Thus using (1.1c) and (3.2), we find that this simplifies to the sought-after identity .

\section{Completion of the proof of theorem 2.1.}

Set $C_{0}=\left(\alpha_{1} \sup _{x \in \bar{\Omega}} \frac{|H|^{2}}{|H . v|}\right)^{2}, C_{1}=\frac{\alpha_{1}}{2} \sup _{x \in \bar{\Omega}}|\operatorname{div} \mathrm{H}|, C_{2}=\frac{\alpha_{1}}{2 \varepsilon} \sup _{x \in \bar{\Omega}}\left|\nabla_{g}(\operatorname{div} H)\right|+\frac{1}{4} \sup _{x \in \bar{\Omega}}|\operatorname{div} H|+$ $\frac{\varepsilon \alpha_{1}}{2} \sup _{x \in \bar{\Omega}}|H(x)|$

From assumptions $(2.1),(2.2)$ and $(2.3)$, we deduce that

$$
\begin{aligned}
& 2 a \int_{S}^{T} \int_{\Omega}\left|\nabla_{g} y\right|_{g}^{2} \mathrm{~d} \Omega d s \leqslant-\int_{S}^{T} \int_{\Gamma_{1}}\left|\nabla_{g} y\right|_{g}^{2} H \cdot v d \Gamma_{1} d s+2 R e \int_{S}^{T} \int_{\Gamma_{1}}\left(\frac{\partial y}{\partial v_{A}}\right) H(\bar{y}) d \Gamma_{1} d s \\
& +\operatorname{Im} \int_{S}^{T} \int_{\Gamma_{1}} y \overline{y_{t}} H \cdot v d \Gamma_{1} d s+\int_{S}^{T} \int_{\Omega}\left|\nabla_{g} y\right|_{g}^{2} \operatorname{div} \operatorname{Hd} \Omega d s \\
& -\left.\operatorname{Im} \int_{\Omega} y H(y) d \Omega\right|_{S} ^{T}-\operatorname{Im} \int_{S}^{T} \int_{\Omega} \overline{y_{t}} y d i v H d Q
\end{aligned}
$$

We also have the estimates, in (3.3c) and (3.3d) $\varepsilon$ is an arbitary positive constant.

$$
\begin{aligned}
& -\int_{S}^{T} \int_{\Gamma_{1}}\left|\nabla_{g} y\right|_{g}^{2} H \cdot v d \Gamma_{1} d s+2 R e \int_{S}^{T} \int_{\Gamma_{1}}\left(\frac{\partial y}{\partial v_{A}}\right) H(\bar{y}) d \Gamma_{1} d s \\
& \leqslant C_{0} \int_{S}^{T} \int_{\Gamma_{1}}\left(\frac{\partial y}{\partial v_{A}}\right)^{2} \mathrm{~d} \Gamma_{1} \mathrm{~d} s 3.3 \mathrm{~b} \\
& \left|\operatorname{Im} \int_{S}^{T} \int_{\Gamma_{1}} y \overline{y_{t}} H \cdot v d \Gamma_{1} d s-\operatorname{Im} \int_{\Omega} y H(y) d \Omega\right|_{S}^{T} \mid \leqslant \\
& \frac{\sup _{x \in \bar{\Omega}}|\mathrm{H}|}{2 \alpha_{1}} \int_{S}^{T} \int_{\Gamma_{1}}|y|^{2} \mathrm{~d} \Gamma_{1} \mathrm{ds}+2 \alpha_{1} \sup _{x \in \bar{\Omega}}|\mathrm{H}| \int_{S}^{T} \int_{\Gamma_{1}}\left|y_{\mathrm{t}}\right|^{2} \mathrm{~d} \Gamma_{1} \mathrm{ds}+\varepsilon \sup _{x \in \bar{\Omega}}|\mathrm{H}(\mathrm{x})| \mathrm{E}(\mathrm{S}) \\
& +\left(\frac{\varepsilon \alpha_{1}}{2} \sup _{x \in \bar{\Omega}}|\mathrm{H}(x)|\right)|y|_{\mathrm{C}\left([\mathrm{S}, \mathrm{T}], \mathrm{L}^{2}(\Omega)\right)}^{2} \\
& \left.\left|\int_{S}^{T} \int_{\Omega}\right| \nabla_{g} y\right|_{g} ^{2} \operatorname{div} \mathrm{Hd} \Omega \mathrm{d} s-\operatorname{Im} \int_{S}^{T} \int_{\Omega} \overline{y_{\mathrm{t}}} y d i v H d \Omega d s \mid \leqslant C_{1} \int_{S}^{T} \int_{\Gamma_{1}}\left(\frac{\partial y}{\partial v_{A}}\right)^{2} \mathrm{~d} \Gamma_{1} d s \\
& +\frac{\varepsilon}{2} \int_{S}^{T} \int_{\Omega}\left|\nabla_{g} \bar{y}\right|_{g}^{2} \mathrm{~d} \Omega \mathrm{ds} \\
& +\left(\frac{\alpha_{1}}{2 \varepsilon} \sup _{x \in \bar{\Omega}}\left|\nabla_{\mathrm{g}}(\operatorname{div} \mathrm{H})\right|+\frac{1}{4} \sup _{x \in \bar{\Omega}}|\operatorname{div} \mathrm{H}|\right) \int_{\mathrm{S}}^{\mathrm{T}} \int_{\Gamma_{1}}|y|^{2} \mathrm{~d} \Gamma_{1} \mathrm{ds}
\end{aligned}
$$




$$
\begin{aligned}
\left|\int_{\Sigma_{1}}\left(\frac{\partial y}{\partial v_{A}}\right)^{2} \mathrm{~d} \Sigma_{1}\right| & \leqslant 3\left[\int_{S}^{T} \int_{\Gamma_{1}}\left(-\int_{0}^{t} k^{\prime}(t-s) y(s) d s\right)^{2} d \Gamma_{1} d s\right. \\
& \left.+\int_{S}^{T} \int_{\Gamma_{1}} k(0)|y|^{2} d \Gamma_{1} d s+\int_{S}^{T} \int_{\Gamma_{1}} b^{2}\left|y_{t}\right|^{2} d \Gamma_{1} d s\right] .
\end{aligned}
$$

Now using a compactness-uniqueness argument in the style of Lasiecka and Triggiani [7], we deduce

$$
|y|_{C\left([S, T], L^{2}(\Omega)\right)}^{2} \leqslant \int_{S}^{T} \int_{\Gamma_{1}}\left|b y_{t}\right|^{2} d \Gamma_{1} d s
$$

Insertion $(3,3 b),(3.3 c),(3.3 d)$ and $(3.3 f)$ in $(3.3 a)$, leads to

$$
\begin{aligned}
& 2 a \int_{S}^{T} \int_{\Omega}\left|\nabla_{g} y\right|_{g}^{2} d \Omega d s \leqslant 3\left(C_{0}+C_{1}\right)\left[\int_{S}^{T} \int_{\Gamma_{1}}\left(-\int_{0}^{t} k^{\prime}(t-s) y(s) d s\right)^{2} d \Gamma_{1} d s\right] \\
& 3\left(C_{0}+C_{1}\right)\left[\int_{S}^{T} \int_{\Gamma_{1}} k(0)|y|^{2} d \Gamma_{1} d s+\int_{S}^{T} \int_{\Gamma_{1}}\left|b y_{t}\right|^{2} d \Gamma_{1} d s\right] \\
& +C_{2} \int_{S}^{T} \int_{\Gamma_{1}}|y|^{2} d \Gamma_{1} d s+\frac{\varepsilon}{2} \int_{S}^{T} \int_{\Omega}\left|\nabla_{g} \bar{y}\right|_{g}^{2} d \Omega d s \\
& +\varepsilon \sup _{x \in \bar{\Omega}}|\mathrm{H}(x)| \mathrm{E}(\mathrm{S})+2 \alpha_{1} \sup _{x \in \bar{\Omega}}|\mathrm{H}| \int_{S}^{T} \int_{\Gamma_{1}}\left|y_{\mathrm{t}}\right|^{2} \mathrm{~d} \Gamma_{1} \mathrm{ds} \\
& +\left(\frac{\varepsilon \alpha_{1}}{2} \sup _{x \in \bar{\Omega}}|\mathrm{H}(\mathrm{x})|\right)|y|_{\mathrm{C}\left([\mathrm{S}, \mathrm{T}], \mathrm{L}^{2}(\Omega)\right)}^{2}
\end{aligned}
$$

Choosing $\varepsilon$ so that $4 a-\varepsilon>0$, we obtain

$$
\begin{aligned}
(4 a-\varepsilon) \int_{S}^{T} E(t) d t \leqslant & {\left[3 C_{3}\left(C_{0}+C_{1}\right)+\varepsilon \sup _{x \in \bar{\Omega}}|H(x)|\right] E(S) } \\
& +2 \alpha_{1} \sup _{x \in \bar{\Omega}}|H| \frac{1}{\sup _{x \in \overline{\Gamma_{1}}}|b|} E(S) \\
& +\left[3\left(C_{0}+C_{1}\right)|k(0)|_{L^{\infty}}^{2}+C_{2}\right] \frac{1}{\digamma} E(S) \\
& +\left[3\left(C_{0}+C_{1}\right)+\frac{\varepsilon \alpha_{1}}{2} \sup _{x \in \bar{\Omega}}|H(x)|\right] \sup _{x \in \bar{\Gamma}_{1}}|b| E(S) .
\end{aligned}
$$

Where

$$
\begin{aligned}
& \int_{S}^{T} E(t) d t \leqslant C E(S) \\
& C= \frac{1}{(4 a-\varepsilon)}\left[3 C_{3}\left(C_{0}+C_{1}\right)+\varepsilon \sup _{x \in \bar{\Omega}}|H(x)|+2 \alpha_{1} \sup _{x \in \bar{\Omega}}|H| \frac{1}{\sup _{x \in \bar{\Gamma}_{1}}|b|}\right. \\
&+\left[3\left(C_{0}+C_{1}\right)+\frac{\varepsilon \alpha_{1}}{2} \sup _{x \in \bar{\Omega}}|H(x)|\right] \sup _{x \in \overline{\Gamma_{1}}}|b| \\
&\left.+\left[3\left(C_{0}+C_{1}\right)|k(0)|_{L^{\infty}}^{2}+C_{2}\right] \frac{1}{\digamma}\right] .
\end{aligned}
$$


Letting $T \rightarrow+\infty$, we obtain for every $S \in \mathbb{R}_{+}$.

$$
\int_{S}^{+\infty} E(t) d t \leqslant C E(S) .
$$

The desired conclusion follows now from Komornik [6].

\section{References}

[1] M. Aassila, M. M. Cavalcanti, et J. A. Soriano, Asymptoic stability and energy deay rate for solution of wave equation with memory in a star shaped domain, SIAM J. Control Optim, 38(2000), 1581-1602. https:/ / doi.org/10.1137/s0363012998344981 1

[2] S. Chai and Y. Guo, Boundary stabilization of wave equations with variable coefficients and memory, Differential and Integral Equations,17(2004), 669-980. 1

[3] R.Cipolotti, E. Machtyngier and San Perdo Siqueira, Nonlinear boundary feedback stabilization for Schrödinger equations, Differrential and Integral Equations, 9(1996), 137-148. 1

[4] A. Guesmia, Thèse de doctorat (Chapitre 8), IRMA, Strasbourg, (2000). 1

[5] E. Hebey, Introduction à l'analyse non linéaire sur les variétés, Editeur Diderot, Paris,(1997).

[6] V. Komornik, Exact controlability and stabilization. The multiplier method. Masson, Paris, (1994). 2, 4

[7] I. Lasiecka and R.Triggiani,Optimal reglarity, exact controllability and uniform stabilization of Schrödinger equations with Dirichlet control,Differrential and Integral Equations, 5(1992), 521-535. 1, 4

[8] J. L. Lions,Quelques méthodes de résolution des problèmes aux limites non linèaires, Dunod, Paris, (1969).

[9] E. Machtyngier and E. Zuazua, Stabilization of Schr ödinger equation, Portugaliae Mathematica, 51 (1994), 243-255.

[10] P. F. Yao, .Observabiliy inequality for exact controllability of wave equations with variable coefficients, SIAM .J. Control Optim, 37(1999), 1568-1599. 1 